\title{
Electrocardiogram Heart Rate as a Predictor of Severity in Acute Alcohol-Related Pancreatitis With Alcohol Withdrawal Syndrome
}

\author{
Stalin Viswanathan ${ }^{1}$, Dheeraj Jain ${ }^{2}$, R Vinayagamoorthi ${ }^{3}$, Murugesan S. Gayathri ${ }^{4}$ \\ 1. General Medicine, Jawaharlal Institute of Postgraduate Medical Education and Research, Pondicherry, IND 2. \\ General Medicine, Indira Gandhi Medical College \& Research Institute, Pondicherry, IND 3. Biochemistry, Indira \\ Gandhi Medical College \& Research Institute, Pondicherry, IND 4. Radiology, Indira Gandhi Medical College \& \\ Research Institute, Pondicherry, IND
}

Corresponding author: Stalin Viswanathan, stalinviswanathan@ymail.com

\section{Abstract \\ Background}

The severity of acute alcohol-related pancreatitis (AAP) with alcohol withdrawal syndrome (AWS) has not been studied. Electrocardiogram (ECG) has not been used as a predictor of severity in patients with AWS and acute pancreatitis.

\section{Objectives}

The study aimed to determine whether the ECG heart rate (HR) could predict the severity of AAP; secondarily, whether AWS influenced the severity of AAP based on Acute Physiology and Chronic Health Evaluation (APACHE) II and Bedside Index for Severity in Acute Pancreatitis (BISAP).

\section{Methods}

Demographics, comorbid illnesses, AWS, biochemistry, ECG, arterial blood gases, and CT findings were noted in patients with AAP. The severity of pancreatitis was scored into mild, moderate, and severe based on CT. BISAP, APACHE II, and ECG heart rate-APACHE (E-APACHE) were compared in patients with and without AWS. A receiver operating characteristic curve was used to find the best predictor of severity.

\section{Results}

Among 138 patients $(\mathrm{M}=128)$, 94 had AWS. ECG changes ( $\geqslant 1)$ were seen in $50 \%$. Patients with AWS were younger, had consumed alcohol for a shorter duration, had higher systemic inflammatory response syndrome (SIRS), APACHE II, and E-APACHE II scores. APACHE II and E-APACHE II correlated significantly with severity grading, HR, alcohol duration, and AWS. HR was the best predictor of severe pancreatitis; EAPACHE was the best predictor for moderately severe pancreatitis.

Received 04/04/2020

Review began 04/06/2020 Review ended 11/17/2020 Published 11/28/2020

\section{(c) Copyright 2020}

Viswanathan et al. This is an open access article distributed under the terms of the Creative Commons Attribution License CC-BY 4.0., which permits unrestricted use, distribution, and reproduction in any medium, provided the original author and source are credited.

\section{Conclusions}

Mostly, AAP appears to be mild; >2/3 ${ }^{\text {rds }}$ have AWS. ECG findings were seen in $50 \%$. HR has not been previously studied in patients with both AAP and AWS and is an easy and inexpensive test to predict the severity of pancreatitis in this cohort.

Categories: Emergency Medicine, Internal Medicine, Gastroenterology

Keywords: alcohol, acute pancreatitis, alcohol withdrawal syndrome, ecg changes, severity scoring

\section{Introduction}

Acute pancreatitis is an inflammatory disorder characterized by acinar cell destruction whose diagnosis is based on the presence of any two of the following: characteristic abdominal pain, elevated lipase or amylase (three times normal), and findings on abdominal imaging [1]. Alcohol withdrawal syndrome (AWS) is a neuropsychiatric syndrome that follows reduction or stopping alcohol [2]. Tachycardia, altered mental status, vomiting, and electrolyte disturbances that are seen in AWS may mimic the clinical features or complications of acute pancreatitis. Arrhythmias, conduction defects, ST-T changes mimicking myocardial infarction, and QT-interval changes have all been described in acute pancreatitis [3]. Only one study of severe acute pancreatitis was available wherein heart rate variability was described to predict complications such as infected necrosis [4]. It was surmised that an electrocardiogram (ECG) would give a more objective and accurate measure of the heart rate than that of the pulse rate measured by the physician at the time of admission. The heart rate is used in both the Acute Physiology and Chronic Health Evaluation (APACHE II) and Bedside Index for Severity in Acute Pancreatitis (BISAP) scores that are commonly used to score the severity in pancreatitis [1]. It was hypothesized that such scoring systems calculated based on the ECG heart 
rate would also differ from those calculated using the pulse rate, and thereby altering the severity scores in patients with acute pancreatitis. So, we embarked on this study to find out whether the ECG heart rate could predict the severity of acute alcohol-related pancreatitis (AAP) in patients with AWS.

\section{Materials And Methods}

\section{Patients}

This retrospective cross-sectional study was performed at Indira Gandhi Medical College and Research Institute, a government-funded teaching institution at Pondicherry, India. The study aimed to find out whether the ECG heart rate could predict the severity of AAP, and secondarily, whether alcohol withdrawal syndrome influenced the severity of AAP based on scoring systems such as APACHE II and BISAP. AAP was diagnosed and classified into mild, moderate, and severe based on the Revised Atlanta Classification 2012 [1]. Alcohol withdrawal syndrome was diagnosed by the presence of any two of the following: autonomic hyperactivity, tremors of the hands, nausea or vomiting, insomnia, transient hallucinations/illusions, anxiety, psychomotor agitation, and generalized tonic-clonic seizures [2]. Records of patients with a diagnosis of acute pancreatitis or acute on chronic pancreatitis admitted between 1 June 2016 and 31 May 2019 were searched for in the Medical Records Department. All patients with a history of alcohol consumption > 6 months and having any two of the following (new-onset acute abdominal pain suggestive of pancreatitis, elevated lipase $>3$ times and contrast-CT findings suggestive of acute pancreatitis) were taken as cases of alcohol-related acute pancreatitis. Patients without a history of alcohol consumption and the presence of cholelithiasis/choledocholithiasis on imaging were excluded from the study. Ethical approval for this study was obtained from the Institute Ethics Committee.

\section{Data collection}

ECGs were perused and the following parameters were noted: heart rate, PR-interval, QRS-interval and QTcintervals, ST-segment changes (elevation or depression), T-wave changes and other abnormalities if any. Heart rate $>100 /$ min was considered as tachycardia, while $\leqslant 60 /$ minute was taken as bradycardia. A short PRinterval was $<120 \mathrm{~ms}$ and prolonged PR-interval was $>200 \mathrm{~ms}$ [5]. QRS-interval $>110 \mathrm{~ms}$ was considered as prolonged [6]. Bazett method was used to calculate $\mathrm{QTc}=\mathrm{QT} /(\sqrt{\mathrm{RR}})$. A QTc $>450 \mathrm{~ms}$ in males and $>460 \mathrm{~ms}$ in females were considered as prolonged QTc [7]. Atrial and ventricular enlargement, poor $r$ wave progression, and low voltage complexes were not considered as ECG changes related to acute pancreatitis. Demographics and history of chronic illnesses such as cirrhosis, chronic kidney disease, surgeries, blood transfusions, diabetes mellitus, and previous episodes of pancreatitis were documented. Alcohol intake, duration and type of beverage, concurrent alcoholic hepatitis and upper gastrointestinal bleed, smoking, past alcohol withdrawal, deaddiction, and Clinical Institute Withdrawal Assessment for Alcohol revised (CIWA-Ar) scores were noted. Pleural effusion on chest radiography, age, altered consciousness, blood urea nitrogen and systemic inflammatory response syndrome $(\geqslant 2)$ were used to calculate the BISAP score [8]. BISAP and APACHE II scores were calculated using Qx Calculate v8.0.3.0 a mobile app. APACHE II scores were calculated using both the admission pulse rate and ECG heart rate (E-APACHE). The findings on abdominal imaging were used to calculate a CT severity index of pancreatitis and patients classified into mild, moderate, and severe pancreatitis based on scores of $0-3,4-6$, and 7-10, respectively. Clinical and laboratory findings were compared between the three groups. Patients were also grouped into those with and without AWS, to find out the association between AWS and AAP.

\section{Statistics}

IBM SPSS for Windows v22 (IBM Corp., Armonk, NY) was used to analyze data. Chi-square test (or Fisher's exact test) was used to analyze categorical variables, while the t-test was used for continuous variables. Pearson's correlation coefficient was calculated for severity grading, ECG heart rate, alcohol duration, and alcohol withdrawal syndrome with respect to APACHE II and BISAP scores. ROC curve was drawn with the three grades of pancreatitis being the state variable, and pulse rate, ECG heart rate, APACHE II and EAPACHE II taken as the test variables. The area under the curve (AUC) was calculated for each severity grade to find the best predictor. A $P$ value of $\leqslant 0.05$ was considered statistically significant.

\section{Results}

There were 138 patients with males constituting 92.8\% ( $\mathrm{n}=128)$. Acute on chronic pancreatitis and alcoholic hepatitis was seen in 43 and 28 patients, respectively. Diabetes mellitus was seen in 31 and seven of these individuals presented with diabetic ketoacidosis. Cirrhosis and chronic kidney disease (CKD) were seen in eight and 12 patients, respectively. Forty-one were smokers. Ninety-four had alcohol withdrawal syndrome, with eight of them had complicated withdrawal (seizures and/or delirium tremens). The CT severity index was available only for 119 patients. ECG changes $(\geqslant 1)$ were seen in $50 \%$. Sinus tachycardia and sinus bradycardia were observed in 31 and 11 patients respectively. Patients with AWS were younger, had consumed alcohol for a shorter duration, had higher systemic inflammatory response syndrome (SIRS), APACHE II, and E-APACHE II score when compared to those without AWS (Table 1). 


\section{Cureus}

\begin{tabular}{|c|c|c|c|}
\hline Gender, Males and females (n) & 90 and 4 & 38 and 6 & 0.04 \\
\hline Age (years) & $41.1 \pm 9.8$ & $44.4 \pm 9.6$ & 0.06 \\
\hline Age $>60(n)$ & 4 & 1 & 0.56 \\
\hline Duration symptoms (d) & $3.8 \pm 4.3$ & $3.2 \pm 2.4$ & 0.43 \\
\hline Duration of stay $(n)$ & $7.2 \pm 2.8$ & $7.8 \pm 2.8$ & 0.20 \\
\hline Ethanol (g) & $90.5 \pm 44.9$ & $84.8 \pm 59.4$ & 0.53 \\
\hline Alcohol duration (years) & $13.7 \pm 7.36$ & $16.5 \pm 7.7$ & 0.04 \\
\hline Smoking (years) & $4.6 \pm 7.3$ & $6.1 \pm 11.2$ & 0.42 \\
\hline Diabetes mellitus (n) & 15 & 16 & 0.007 \\
\hline Previous admissions $(\mathrm{n})$ & 46 & 15 & 0.10 \\
\hline Pulse rate (beats/min) & $84.2 \pm 12.6$ & $84.7 \pm 17.1$ & 0.85 \\
\hline Mean arterial pressure $(\mathrm{mmHg})$ & $93 \pm 14.0$ & $90.1 \pm 15.0$ & 0.28 \\
\hline Respiratory rate (breaths/min & $20.7 \pm 5.0$ & $19.6 \pm 6.2$ & 0.26 \\
\hline Palpable Liver (n) & 21 & 3 & 0.02 \\
\hline Total bilirubin mg/dL & $1.9 \pm 1.5$ & $2.3 \pm 3.4$ & 0.37 \\
\hline Aspartate aminotransferase IU/L & $90 \pm 92.2$ & $83.8 \pm 113.1$ & 0.75 \\
\hline Alanine aminotransferase IU/L & $68.0 \pm 114.9$ & $47.8 \pm 49.7$ & 0.32 \\
\hline Alkaline phosphatase IU/L & $173.9 \pm 128.8$ & $248.2 \pm 283.2$ & 0.19 \\
\hline Protein $\mathrm{g} / \mathrm{dL}$ & $8.9 \pm 2.1$ & $6.5 \pm 1.0$ & 0.50 \\
\hline Prothrombin time prolongation (s) & $12.1 \pm 28.9$ & $16.4 \pm 7.8$ & 0.06 \\
\hline Lipase (U/L) & $403 \pm 216.9$ & $405 \pm 289.1$ & 0.96 \\
\hline Maddrey discriminant function & $29.2 \pm 16.6$ & $41.0 \pm 20.7$ & 0.09 \\
\hline Hematocrit \% & $39.5 \pm 6.5$ & $38.3 \pm 11.2$ & 0.53 \\
\hline Total leukocyte count $\times 10^{9} / \mathrm{L}$ & $9.962 .7 \pm 4.131 .2$ & $8.978 \pm 4.211 .0$ & 0.20 \\
\hline ECG heart rate /min & $85.4 \pm 20.2$ & $82.0 \pm 16.9$ & 0.30 \\
\hline ECG tachycardia $(n)$ & 22 & 9 & 0.69 \\
\hline ECG bradycardia (n) & 8 & 3 & 0.73 \\
\hline ECG PR interval & $139.3 \pm 21.4$ & $138.7 \pm 21.5$ & 0.89 \\
\hline Short PR interval $<120 \mathrm{~ms}(\mathrm{n})$ & 17 & 9 & 0.74 \\
\hline ECG QTc interval & $427.9 \pm 27.7$ & $427.6 \pm 23.4$ & 0.94 \\
\hline Prolonged QTc interval (n) & 17 & 8 & 0.98 \\
\hline Mild pancreatitis (n) & 49 & 22 & 0.81 \\
\hline Moderate pancreatitis (n) & 23 & 12 & 0.72 \\
\hline Severe pancreatitis $(n)$ & 9 & 4 & 0.92 \\
\hline Acute on chronic pancreatitis (n) & 14 & 29 & 0.90 \\
\hline Systemic inflammatory response syndrome (n) & 26 & 19 & 0.07 \\
\hline BISAP 0-2 (n) & 92 & 43 & 0.95 \\
\hline APACHE II score & $5.5 \pm 3.7$ & $7.5 \pm 3.9$ & 0.04 \\
\hline APACHE II ECG & $5.9 \pm 3.5$ & $8.6 \pm 3.9$ & 0.009 \\
\hline
\end{tabular}




\section{Cureus}

\begin{tabular}{|c|c|c|c|}
\hline Infection (n) & 3 & 3 & 0.33 \\
\hline Diabetic acidosis (n) & 3 & 4 & 0.14 \\
\hline Upper gastrointestinal bleed (n) & 4 & 3 & 0.53 \\
\hline Acute kidney injury (n) & 10 & 3 & 0.43 \\
\hline Alcoholic hepatitis (n) & 21 & 7 & 0.38 \\
\hline Intensive care unit stay (n) & 9 & 4 & 0.92 \\
\hline Death (n) & 0 & 4 & 0.03 \\
\hline
\end{tabular}

TABLE 1: Baseline characteristics, laboratory parameters, and complications in those with and without alcohol withdrawal syndrome

AWS, alcohol withdrawal syndrome; APACHE, Acute Physiology and Chronic Health Evaluation; ECG, electrocardiogram; BISAP, Bedside Index for Severity in Acute Pancreatitis

The patient's age and mean arterial pressure, APACHE and E-APACHE score, BUN, creatinine and acute kidney injury (AKI), and ECG PR-interval varied significantly among the various severity grades of pancreatitis (Table 2).

\begin{tabular}{|c|c|c|c|c|}
\hline$N=119 / 138$ & Mild $(n=71)$ & Moderate (n=35) & Severe $(n=13)$ & Significance \\
\hline Age (years) & $40.8 \pm 10.1$ & $43.3 \pm 9.4$ & $49.2 \pm 8.7$ & 0.01 \\
\hline Age >60 (n) & 2 & 1 & 2 & 0.10 \\
\hline Duration of symptoms (d) & $4.0 \pm 9.4$ & $3.2 \pm 1.19$ & $2.8 \pm 1.9$ & 0.46 \\
\hline Duration of Stay (d) & $7.4 \pm 2.8$ & $8.1 \pm 2.9$ & $7.2 \pm 2.5$ & 0.47 \\
\hline Alcohol duration (years) & $13.6 \pm 7.3$ & $17.7 \pm 8.0$ & $12.9 \pm 8.1$ & 0.02 \\
\hline Ethanol (g) & $87.1 \pm 54.2$ & $88.3 \pm 49.1$ & 84.341 .2 & 0.97 \\
\hline Smoking (years) & $4.8 \pm 7.5$ & $4.3 \pm 9.2$ & $6.5 \pm 13.1$ & 0.16 \\
\hline Diabetes mellitus (n) & 20 & 3 & 5 & 0.03 \\
\hline Cirrhosis (n) & 2 & 4 & 2 & 0.24 \\
\hline Past history pancreatitis (n) & 21 & 7 & 1 & 0.18 \\
\hline Temperature (F) & $98.8 \pm 1.3$ & $98.3 \pm 1.3$ & $98.9 \pm 1.4$ & 0.63 \\
\hline Pulse rate (beats/min) & $83.2 \pm 15.8$ & $85.5 \pm 10.8$ & $87.4 \pm 16.4$ & 0.49 \\
\hline Respiratory rate (breaths/ min) & $20.1 \pm 6.3$ & $20.0 \pm 4.7$ & $21.2 \pm 5.4$ & 0.76 \\
\hline Mean arterial pressure $(\mathrm{mmHg})$ & $89.9 \pm 14.2$ & $92.5 \pm 9.0$ & $86.3 \pm 12.9$ & 0.09 \\
\hline Hematocrit(\%) & $39.7 \pm 8.6$ & $36.6 \pm 7.9$ & $37.8 \pm 7.5$ & 0.18 \\
\hline Total leukocyte count (x109/L) & $9.457 \pm 3.871$ & $9.722 \pm 4.368$ & $11.361 \pm 6.163$ & 0.34 \\
\hline Systemic inflammatory response syndrome (n) & 22 & 10 & 7 & 0.22 \\
\hline BISAP Median score & 4 & 8 & 6 & \\
\hline CT Severity index (Median) & 1 & 2 & 4 & $<0.001$ \\
\hline APACHE II score & $4.3 \pm 2.9$ & $7.5 \pm 3.8$ & $9.5 \pm 3.5$ & $<0.001$ \\
\hline APACHE II ECG-Heart rate score & $5.1 \pm 3.1$ & $8.13 \pm 3.9$ & $9.5 \pm 2.9$ & 0.001 \\
\hline Blood urea nitrogen & $4.3 \pm 1.6$ & $6.46 \pm 5.3$ & $7.67 \pm 4.6$ & 0.01 \\
\hline Creatinine(mg/dL) & $0.9 \pm 0.1$ & $1.4 \pm 1.1$ & $1.5 \pm 0.8$ & 0.002 \\
\hline
\end{tabular}




\section{Cureus}

\begin{tabular}{|c|c|c|c|c|}
\hline Acute kidney injury (n) & 1 & 8 & 3 & 0.001 \\
\hline Upper gastrointestinal bleed (n) & 5 & 2 & 0 & 0.60 \\
\hline Infection (n) & 3 & 3 & 0 & 0.43 \\
\hline Diabetic ketoacidosis (n) & 4 & 2 & 0 & 0.68 \\
\hline Bilirubin (mg/dL) & $1.8 \pm 1.4$ & $1.9 \pm 1.5$ & $3.2 \pm 4.7$ & 0.12 \\
\hline Aspartate aminotransferase (U/L) & $83.4 \pm 105.9$ & $78.3 \pm 81.1$ & $114.8 \pm 120.2$ & 0.54 \\
\hline Alanine aminotransferase (U/L & $51.4 \pm 49.1$ & $77.0 \pm 175.4$ & $51.2 \pm 37.6$ & 0.51 \\
\hline Prothrombin time prolongation(s) & $14.1 \pm 8.6$ & $9.9 \pm 8.4$ & $11.8 \pm 5.3$ & 0.20 \\
\hline Maddrey discriminant function score & $35.0 \pm 20.9$ & $24.7 \pm 13.5$ & $47.2 \pm 19.2$ & 0.08 \\
\hline Alcoholic hepatitis (n) & 10 & 11 & 4 & 0.07 \\
\hline Severe alcoholic hepatitis (n) & 5 & 4 & 2 & 0.52 \\
\hline Alcohol withdrawal syndrome (n) & 49 & 23 & 9 & 0.93 \\
\hline CIWA score & 17 & 9 & 3 & 0.89 \\
\hline Complicated withdrawal (n) & 4 & 2 & 1 & 0.95 \\
\hline Chronic calcific pancreatitis ( $\mathrm{n}$ ) & 3 & 0 & 0 & 0.35 \\
\hline Pancreatic Ascites(n) & 0 & 5 & 6 & 0.001 \\
\hline Death (n) & 0 & 1 & 3 & $<0.001$ \\
\hline ECG heart rate & $84.6 \pm 20.0$ & $83.9 \pm 19.4$ & $86.7 \pm 21.0$ & 0.91 \\
\hline ECG tachycardia (n) & 17 & 6 & 4 & 0.56 \\
\hline ECG PR & $135.7 \pm 20.4$ & $143.8 \pm 21.1$ & $150.3 \pm 22.7$ & 0.02 \\
\hline Short PR (n) & 16 & 5 & 1 & 0.33 \\
\hline ECG QRS & $92.0 \pm 10.5$ & $90.7 \pm 9.9$ & $89.7 \pm 8.0$ & 0.67 \\
\hline ECG QTC & $423.6 \pm 26.2$ & $429.2 \pm 27.7$ & $439.1 \pm 28.2$ & 0.14 \\
\hline Pro & 10 & 5 & 4 & 0.30 \\
\hline
\end{tabular}

TABLE 2: Clinical characteristics and laboratory investigations in patients with various grades of pancreatitis

CIWA, Clinical Institute Withdrawal Assessment; ECG, electrocardiogram; BISAP, Bedside Index for Severity in Acute Pancreatitis; APACHE, Acute Physiology and Chronic Health Evaluation

APACHE II and E-APACHE II score correlated significantly with the severity grading, ECG heart rate, alcohol duration, and alcohol withdrawal syndrome (Table 3). 


\section{Cureus}

\begin{tabular}{|c|c|c|}
\hline Variables & Correlating factors & Correlation; Significance (Cl) \\
\hline \multirow{6}{*}{ Severity grading } & APACHE II & $\mathrm{R}=0.502 ; \mathrm{P}<0.001(0.293-0.691)$ \\
\hline & E-APACHE II & $\mathrm{R}=0.448 ; \mathrm{P}<0.001(0.180-0.663)$ \\
\hline & BISAP score & $\mathrm{R}=0.348 ; \mathrm{P}<0.001(0.209-0.611)$ \\
\hline & Alcoholic hepatitis & $R=0.186 ; P<0.04(0.004-0.239)$ \\
\hline & Patient age & $\mathrm{R}=0.243 ; \mathrm{P}<0.004(0.100-0.404)$ \\
\hline & Death & $\mathrm{R}=0.337 ; \mathrm{P}<0.001(0.191-0.543)$ \\
\hline \multirow{2}{*}{ ECG heart rate } & APACHE II & $R=0.357 ; P=0.03(0.058-0.632)$ \\
\hline & BISAP & $R=0.375 ; P=0.02(0.018-0.703)$ \\
\hline \multirow{3}{*}{ Alcohol duration (years) } & ECG heart rate & $R=0.332 ; P=0.04(0.033-0.564)$ \\
\hline & APACHE II & $\mathrm{R}=0.345 ; \mathrm{P}=0.03(0.053-0.593)$ \\
\hline & E-APACHE II & $\mathrm{R}=0.357 ; \mathrm{P}=0.03(0.058-0.632)$ \\
\hline & APACHE II & $R=-0.243 ; P=0.04(-0.523-0.44)$ \\
\hline ol withdr & E-APACHE & .318 \\
\hline
\end{tabular}

TABLE 3: Correlation of severity grading, ECG heart rate, alcohol duration and alcohol withdrawal syndrome

ECG, electrocardiogram; BISAP, Bedside Index for Severity in Acute Pancreatitis; APACHE, Acute Physiology and Chronic Health Evaluation

ECG heart rate was the best predictor of severe pancreatitis with AUC of 0.773 (for a cut-off of 90 beats/min, with a sensitivity and specificity of $71.5 \%$ and $74.5 \%$ respectively) while E-APACHE was the best predictor for moderately severe pancreatitis (Table 4 and Figure 1).

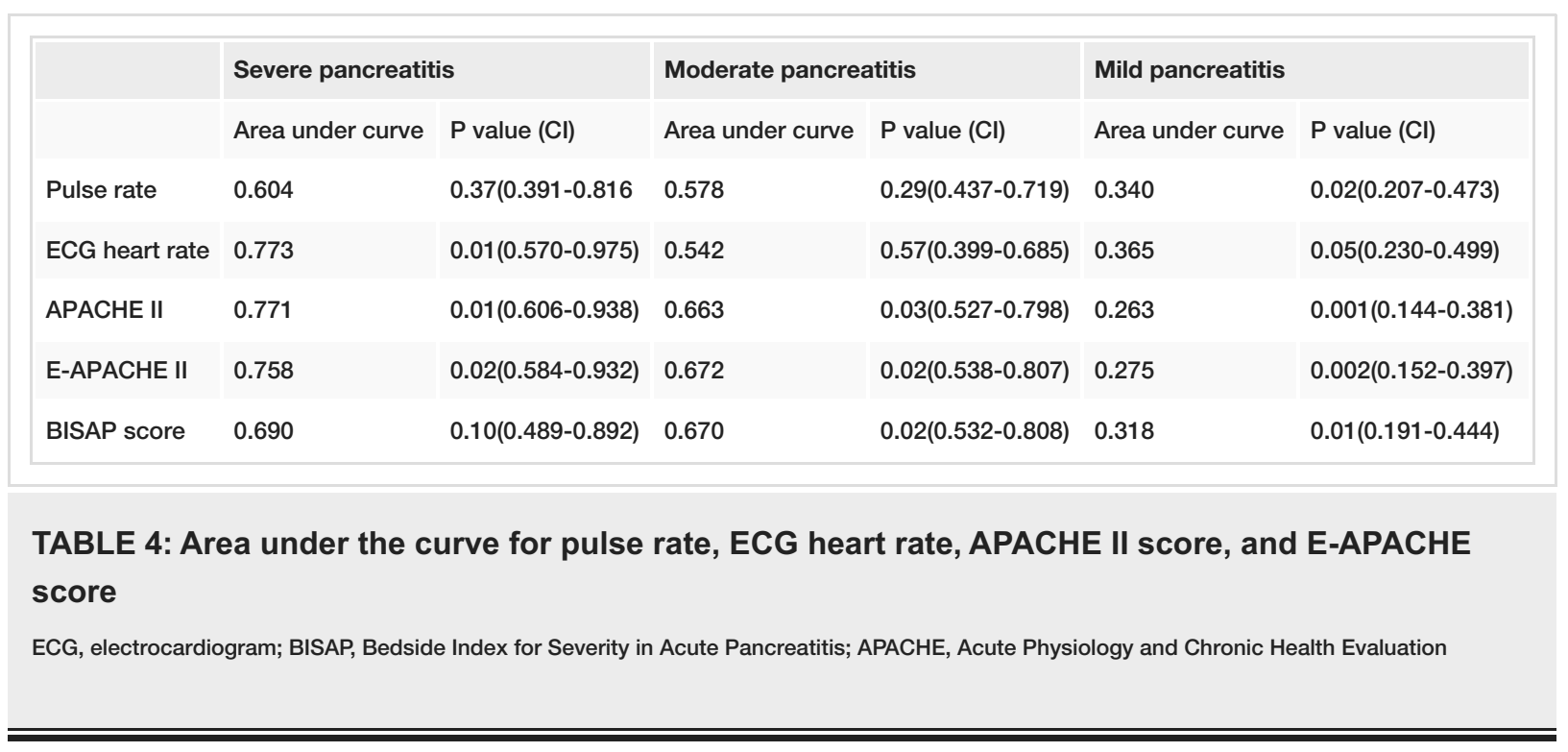



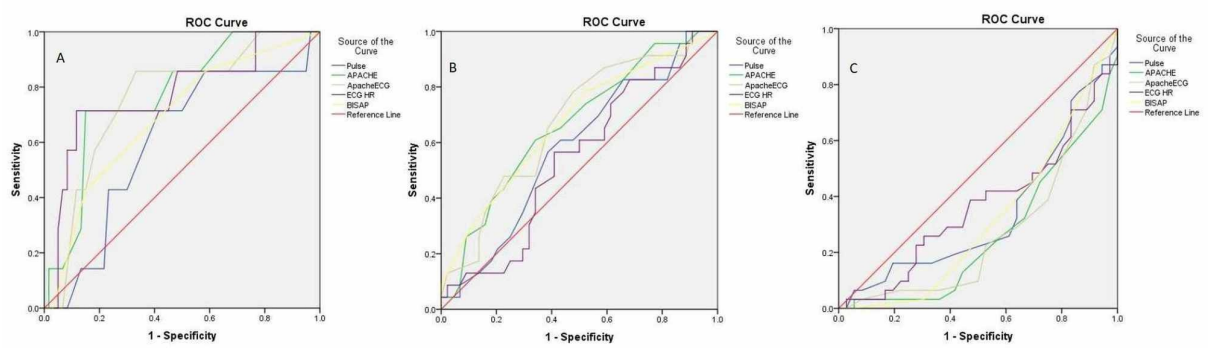

FIGURE 1: Area under the curve for severe (A), moderate (B), and mild (C) acute alcohol-related pancreatitis

\section{Discussion}

In most countries, alcohol is the second commonest cause of acute pancreatitis [9]. In developing countries, AAP causes about 30\% of cases [10]. Gallstone-related pancreatitis is twice as common as AAP [11]. Studies from India report either alcohol or gall stones as the commonest reason for pancreatitis. Alcohol was the commonest cause for acute pancreatitis in studies by $50 \%(n=54)$ by Chauhan et al., $59.63 \%(n=123)$ by Negi et al., and $51 \%(\mathrm{n}=110)$ by Vengadakrishnan et al. [12-14]. In a study from Kolkata of 234 patients, AAP constituted only $29 \%$ [15]. Their study had $63.5 \%$ with diabetes mellitus and $56.2 \%$ smokers compared to $22.5 \%$ and $29.5 \%$ in our study respectively. APACHE and BISAP scores were not calculated in any of these studies, nor was AWS a complication reported in either study. In a study by Venkatesh et al., a comparison of scoring systems in 164 patients (115 with AAP) was studied, but without the component of alcohol withdrawal [16]. Only 23\% (40/168) of patients in a study from Delhi was due to AAP [17].

Friedreich first proposed the relation between alcohol and pancreatitis in 1878 [18]. Only a minority of patients drinking alcohol will develop pancreatitis, indicating a second susceptibility factor. The drinking pattern and diet are not associated with the development of pancreatitis. Obesity and inherited factors such as SPINK1 mutations have a proven association. Smoking and type of beverage have been shown/not shown to have an association in different studies [19]. Forty-one patients in our study were smokers;17/25 patients with acute on chronic pancreatitis were smokers. We did not have details regarding the diet and BMI of our patients.

About $50 \%$ of patients develop alcohol withdrawal on stopping or reducing alcohol [20]. Sixty-eight percent of our patients had withdrawal at or following admission into hospital. Coexisting gastrointestinal or respiratory disease are risk factors for withdrawal delirium. Alcohol withdrawal syndrome is characterized by tachycardia, hypertension, altered consciousness (delirium), dyselectrolytemia (potassium and sodium), and hemoconcentration (vomiting), all of which can affect severity scoring systems such as APACHE II and BISAP [20]. APACHE II and E-APACHE II scores were significantly lower in patients with AWS compared to those without (Table 1). We believe that could partly be due to younger patients, and hemodilution and anemia (CKD, cirrhosis, alcoholic hepatitis, upper gastrointestinal bleed, acute on chronic pancreatitis, and alcohol). None of the four deaths had AWS. Only one study was available that explored the relationship between AAP and alcohol withdrawal [21]. Nordback et al. hypothesized that pancreatitis was one of the manifestations occurring due to withdrawal; they found that $71 \%(\mathrm{n}=100)$ developed pancreatitis during the first two days of withdrawal. We did not have data regarding the time of alcohol withdrawal in relation to pancreatitis [21].

The 2012 Revised Atlanta Classification is the most used system to grade pancreatitis [1]. Based on the CTSI, the frequencies among the 138 patients were $58(0-1), 37(2-3), 16(4-6)$, and 1 (8-10). Three patients with CTSI of 4-6 and one patient with CTSI of 8 died in our study. Necrosis and pseudocyst were seen in three patients each, while ascites and pleural effusion were seen in 11 and 9 patients respectively. BISAP and APACHE II scores are commonly used to predict the severity of acute pancreatitis, the former due to its accuracy and simplicity, and the latter due to it being highly validated [1]. The accuracy of various scoring systems is comparable [9]. Eighty to $85 \%$ of patients with acute pancreatitis have mild severity that corresponded with $76 \%$ in our study [1]. In our study, APACHE II and E-APACHE-II scores did well for all the grades of pancreatitis, while BISAP had high AUC only for moderate and mild pancreatitis (Table 4).

Hypothesized reasons for myocardial involvement in pancreatitis include dyselectrolytemia, toxic effects of pancreatic enzymes, and a cardiobiliary reflex among others [22]. Cardiovascular changes in acute pancreatitis include tachycardia, hypovolemia, cardiac regional wall motion abnormalities, pericardial effusion, ventricular and atrial fibrillation, and QTc prolongation, shortened PR interval, bradycardia, STsegment depression and elevation, and T-wave changes [18]. Only PR-interval was significant between the three groups of pancreatitis, with mild pancreatitis having significantly shorter PR-intervals (135.6 $\pm 20.4 \mathrm{vs}$ $142.8 \pm 21.8$ ) compared to the others. Fifty percent of patients with pancreatitis can have ECG changes. Nadkarni et al. prospectively studied 50 patients without prior cardiac illness and found that prolonged QTc 
and diastolic dysfunction were predictors of mortality in acute pancreatitis. All patients who died had prolonged QTc [23]. In our study, sinus tachycardia, sinus bradycardia, prolonged QTc, and short PR-interval were seen in $31,11,25$, and 26 individuals respectively. Among the four deaths in our study, one had sinus tachycardia and prolonged QTc each, and none had a short PR interval or sinus bradycardia. None of the four had dyselectrolytemia that could contribute to a prolonged QTc. T-wave changes were seen in three (including one Wellen's pattern), and ST-segment changes were observed in two patients. Echocardiography could not be done in all patients, but in the seven that were performed none had pericardial effusion or diastolic dysfunction.

In a study from Turkey, ECG was studied in 64 patients with acute pancreatitis and $65 \%$ of patients had at least one electrolyte abnormality, the most common being hypokalemia [24]. In another study of 54 patients with severe acute pancreatitis and ECG findings, hypomagnesemia $(n=15)$ was an important negative correlate of sinus tachycardia $(n=15)$. A third of their patients had elevated CK-MB [25]. The ECG was not directly correlated with the severity scoring system. Mehmet and colleagues studied QT intervals and QT dispersion in 134 patients with acute pancreatitis, 32 of them related to alcohol. Eighty-eight of their patients had changes in ECG, with early repolarization in lateral leads being the most common finding. QT intervals were not prolonged in their patients [26]. QT interval dispersion (the difference between the maximum and minimum QT intervals) was significant between the ECGs performed during an acute attack of pancreatitis and at the time of remission [26]. In another study of 120 patients from Turkey who presented with an acute abdomen, the performance of an ECG resulted in a diagnosis needing management by the Cardiology services in 10 patients. Overall, 38 patients had ECG changes and one died [27].

Heart rate (variability) has been studied only once in severe acute pancreatitis in 41 patients (seven with AAP) with pancreatic necrosis and multiorgan dysfunction syndrome [4]. Features suggestive of sympathetic suppression during the acute phase predicted these two complications with a high AUC. Our study is the first to study heart rate as a predictor of the severity of AAP in the presence of AWS. ECG heart rate could not predict complications such as ascites, necrosis or pseudocyst formation in our study.

\section{Limitations}

Our study had some limitations. Triglycerides and calcium were not available for all patients and hence hypertriglyceridemia as an etiological factor could not be excluded. Facility for lactate dehydrogenase was unavailable and hence Ranson scoring could not be performed. Arterial blood gases and CT abdominal findings were available only in 69/138 and 113/138 patients respectively. Detailed drug history was available for only one patient with hydrochlorthiazide use as a contributing factor for pancreatitis. Being a teaching hospital, there was an element of referral bias contributing to the number of patients with pancreatitis. This retrospective data was from a single treating Unit in the Department of Medicine. We did not access data of the other four medical units and the Surgery department which may have shown a different picture regarding the severity and mortality of AAP.

\section{Conclusions}

Most patients with AAP appear to have mild severity of pancreatitis and more than two-thirds are associated with an alcohol withdrawal syndrome. ECG findings in AAP are also seen in half the patients, comparable to other studies of acute pancreatitis. ECG heart rate has not been previously studied in patients with both AAP and AWS and is an easy and inexpensive test to predict the severity of pancreatitis in this cohort.

\section{Additional Information \\ Disclosures}

Human subjects: Consent was obtained by all participants in this study. Institute Ethics Committee issued approval IEC/PP/2015/42. Animal subjects: All authors have confirmed that this study did not involve animal subjects or tissue. Conflicts of interest: In compliance with the ICMJE uniform disclosure form, all authors declare the following: Payment/services info: All authors have declared that no financial support was received from any organization for the submitted work. Financial relationships: All authors have declared that they have no financial relationships at present or within the previous three years with any organizations that might have an interest in the submitted work. Other relationships: All authors have declared that there are no other relationships or activities that could appear to have influenced the submitted work.

\section{References}

1. Leppäniemi A, Tolonen M, Tarasconi A, et al.: 2019 WSES guidelines for the management of severe acute pancreatitis. World J Emerg Surg. 2019, 14:27. 10.1186/s13017-019-0247-0

2. Finnell JT: Alcohol-Related Disease. In: Walls R, Hockberger R, Gausche-Hill M, eds . Rosen’s Emergency Medicine: Concepts and Clinical Practice. 9th, 142:1838-1851.

3. Kumar AV, Mohan Reddy G, Anirudh Kumar A: Acute pancreatitis complicated by acute myocardial infarction: a rare association. Indian Heart J. 2013, 65:474-477. 10.1016/j.ihj.2013.06.009

4. Zhang L, Zhou J, Ke L, et al.: Role of heart rate variability in predicting the severity of severe acute 
pancreatitis. Dig Dis Sci. 2014, 59:2557-2564. 10.1007/s10620-014-3192-5

5. Parry-Williams G, Malhotra A, Dhutia H, et al.: The short PR interval in young athletes . Heart. 2019, 105:2526. 10.1136/heartjnl-2019-BCS.28

6. Goldberger AL, Goldberger ZD, Shvilkin A: Chapter 3. How to Make Basic ECG Measurements. Goldberger's Clinical Electrocardiography. 9th Ed. New York:Elsevier. 2018, 11:20. 10.1016/B978-0-323-40169-2.00003-2

7. QT interval and drug therapy. BMJ. 2016, 353:2732. 10.1136/bmj.i2732

8. Olson E, Perelman A, Birk JW: Acute management of pancreatitis: the key to best outcomes . Postgrad Med J. 2019, 95:328-333. 10.1136/postgradmedj-2018-136034

9. Van Dijk SM, Hallensleben NDL, Van Santvoort HC, et al.: Acute pancreatitis: recent advances through randomised trials. Gut. 2017, 66:2024-2032. 10.1136/gutjnl-2016-313595

10. Wang GJ, Gao CF, Wei D, et al.: Acute pancreatitis: etiology and common pathogenesis . World J Gastroenterol. 2009, 15:1427-1430. 10.3748/wjg.15.1427

11. Tandon RK: Chapter 59. Management of Acute Pancreatitis: Indian Guidelines and Protocols. In: Muruganathan A. Ed. Medicine Update. Volume 23. New Delhi: Jaypee Brothers Medical Publishers. 2013 p, 267:70. http://apiindia.org/wp-content/uploads/medicine_update_2013/chap59.pdf

12. Chauhan $\mathrm{Y}$, Jindal N, Verma R, et al.: A clinical profile and outcome of patients with acute pancreatitis: a prospective study in North India. Arch Int Surg. 2018, 8:132-138. 10.4103/ais.ais_3_19

13. Negi N, Mokta J, Sharma B, et al.: Clinical profile and outcome of acute pancreatitis: a hospital-based prospective observational study in sub Himalayan state. J Assoc Physicians India. 2018, 66:22-24.

14. Vengadakrishnan K, Koushik AK: A study of the clinical profile of acute pancreatitis and its correlation with severity indices. Int J Health Sci. 2015, 9:411-417.

15. Mukherjee D, Bhakta S, Lahiry S, et al.: Demographic profile of acute pancreatitis in Eastern India: a single centre experience. Asian J Med Sci. 2017, 8:24-29. 10.3126/ajms.v8i6.18282

16. Venkatesh NR, Vijayakumar C, Balasubramaniyan G, et al.: Comparison of different scoring systems in predicting the severity of acute pancreatitis: a prospective observational study. Cureus. 2020, 12:6943. 10.7759/cureus.6943

17. Garg P, Khanna S, Bohidar N, et al.: Incidence, spectrum and antibiotic sensitivity pattern of bacterial infections among patients with acute pancreatitis. J Gastroenterol Hepatol. 2001, 16:1055-1059. 10.1046/j.1440-1746.2001.02589.x

18. Yegneswaran B, Kostis JB, Pitchumoni CS: Cardiovascular manifestations of acute pancreatitis . J Crit Care. 2011, 26:225.e11-225.e18. 10.1016/j.jcrc.2010.10.013

19. Apte M V., Pirola RC, Wilson JS: Mechanisms of alcoholic pancreatitis. J Gastroenterol Hepatol. 2010, 25:1816-1826. 10.1111/j.1440-1746.2010.06445.x

20. Pace C: Alcohol withdrawal: Epidemiology, clinical manifestations, course, assessment, and diagnosis. In Saitz R, ed. UptoDate; 2020[cited 28 Feb2020]. 28:2020.

21. Nordback I, Pelli H, Lappalainen-Lehto R, Sand J: Is it long-term continuous drinking or the post-drinking withdrawal period that triggers the first acute alcoholic pancreatitis?. Scand J Gastroenterol. 2005, 40:12351239. 10.1080/00365520510023413

22. Hsu PC, Lin TH, Su HM, et al.: Acute necrotizing pancreatitis complicated with ST elevation acute myocardial infarction: a case report and literature review. Kaohsiung J Med Sci. 2010, 26:200-205. 10.1016/S1607-551X(10)70029-2

23. Nadkarni N, Bhasin DK, Rana SS, et al.: Diastolic dysfunction, prolonged QTc interval and pericardial effusion as predictors of mortality in acute pancreatitis. J Gastroenterol Hepatol. 2012, 27:1576-1580. 10.1111/j.1440-1746.2012.07229.x

24. Yaylaci S, Kocayigit I, Genc A., et al.: Electrocardiographic changes in patients with acute pancreatitis . Med J DY Patil Univ. 2015, 8:196-198. 10.4103/0975-2870.153159

25. Huang LY, Ma BW, He F, et al.: Electrocardiographic, cardiac enzymes, and magnesium in patients with severe acute pancreatitis. Gastroenterol Nurs. 2012, 35:256-260. 10.1097/SGA.0b013e31826092a6

26. Buğdacı MS, Tüzün A, Koca H, et al.: QT interval changes and reversibility of QT dispersion in patients with acute pancreatitis. Turk J Gastroenterol. 2014, 25:59-62. 10.5152/tjg.2014.4960

27. Oguzturk H, Turtay MG, Tekin YK, et al.: The evaluation of electrocardiogram findings in acute abdominal pain patients admitted to the emergency department. J Prim Care Community Health. 2011, 2:163-166. $10.1177 / 2150131911403931$ 